\title{
Structure Design of Multi-function Ball Screw Measuring Device and Research on Testing Scheme
}

\author{
Haitao $\mathrm{Li}^{\mathrm{a}}$, Junjie Guo ${ }^{\mathrm{b}}$, Hongfeng $\mathrm{Du}^{\mathrm{c}}{ }^{\mathrm{C}}$, Xing Wang ${ }^{\mathrm{d}}$ \\ School of Mechanical Engineering, Xi'an Jiaotong University, Xi'an 710049, China \\ apirate328@163.com, bjunjieg520@163.com, ${ }^{\mathrm{c}} \mathrm{dhfmailbox@163.com,}{ }^{\mathrm{d}} 67$ wangxing@163.com
}

Keywords: ball screw; geometric accuracy; friction torque; preload; fatigue properties; temperature rise.

\begin{abstract}
A multi-function ball screw measuring device is developed, which can achieve most of the parameter testing of ball screw like geometric accuracy, positional accuracy, reverse clearance, speed and acceleration, friction torque, preload, fatigue properties, temperature rise and so on. According to the supporting features of parameters testing, we can divide the test bed into two categories: double dead center test bed and double bearing seat test bed. The former is used to do low speed and high precision test experiments, and the latter is used to do high speed and heavy load test experiment. By the means of reasonable structure design and simply assembly, we can conduct these two kinds of test on the ball screw measuring device. In this way, not only the cost is cut down, but also the comprehensive and reliable evaluation for ball screw will be obtained. In this paper, detail introduction of test platform building and test program for different test parameters is given. The content of paper is divided into two parts, the first part describes the overall structure design, double dead center test platform building and geometric accuracy test program, the second part describes double bearing block test platform building and relevant test programs.
\end{abstract}

\section{Introduction}

As a one of the key components in mechanical transmission, ball screw assembly has major functions: transform rotary motion to linear motion or transform torque to axial force. It has been widely used in the field of aircraft mechanics, CNC machine tools and precise instruments because of the characters of high precision, reversibility and high efficiency. Since our country developed the first ball screw assembly in 1964, the manufacturing of it has been greatly improved. But there is still a large gap in quality of the products compared with some developed countries. That is because the test instruments are not complete, some key performance indicators fail to be tested. Moreover, loss of unified inspection procedures in mechanical industry hinders the development of theoretical research and production practice ${ }^{[1-4]}$.

At present, domestic scholars have done vast majority of research work, various ball screw detection equipment have been developed ${ }^{[1,2]}$. Feng Hu-tian and Yin Ai-hua coming from Nanjing University of Science has carried out fruitful research in ball screw measurement, and ball screw pitch error measuring device and comprehensive performance test equipment are developed. The ball screw test equipment GSZ2000 specially developed by Beijing Machine Tool Research Institute can test ball screw performance at high speed. But they need several equipments to test the geometric accuracy and high speed dynamic performance, so the test costs are high. In this paper, we develop s new detection equipment. By the means of reasonable structure design and simply assembly, we can conduct these two kinds of test using this equipment. In this way, not only the cost is cut down, but also the comprehensive and reliable evaluation for ball screw will be obtained. Meanwhile, a new principle of ball screw helical line error measurement is proposed.

\section{Overall introduction of multi-function ball screw measuring device [1, 2]}

The multi-function ball screw measuring device can measure a ball screw which has maximum length 
of $2200 \mathrm{~mm}$, maximum effective length of $2000 \mathrm{~mm}$, diameter vary from $\varphi 20$ to $\varphi 100$. The maximum designed speed is $3000 \mathrm{r} / \mathrm{min}$, measuring accuracy is $1 \mathrm{um}$, maximum linear velocity is $60 \mathrm{~m} / \mathrm{min}$, the spindle radial run-out and face run-out are less than $1 \mathrm{um}$. The structural component of the test bed is showed in figure 1.

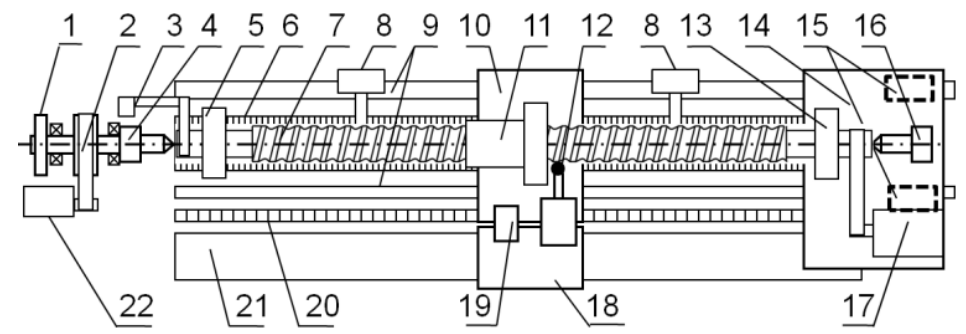

1 circular grating encoder 2 synchronous belt 3 fork 4 precision spindle 5 front bearing seat 6 linear motor 7 ball screw 8 cantilevers 9 linear guide ways 10 linear guide slider 11 ball screw nut

12 measuring head 13 back bearing seat 14 tailstock slider 15 pneumatic clamps16 tailstock 17 AC servo motor 18 air flotation slider 19 linker 20 long grating encoder 21 aerostatic guide way 22 torque motor 23 nut seat

Figure 1 overall structure design diagram of measuring instrument

As it shows in figure 1 , the base body of measuring instrument is made of granite. The aerostatic guide 21 and linear guide way 9 are parallel-arranged on the base body. Air flotation slider 18 with the whole measuring head frame mounted on is set on the aerostatic guide 18 and is able to move in an axial direction. Linear guide slider 10 and tailstock slider 14 which are all set on linear guide way 9 can move along the axis. There is a linker 19 between linear guide slider 10 and air flotation slider 18 . The tailstock 16 is mounted on tailstock slider 14. There are two Hema pneumatic clamps 15 set under the tailstock slider 14 to fix the tailstock slider 14 on linear guide way 9 at any position.

The back bearing seat 13 is mounted on tailstock slider 14 , so it can move along the axis with tailstock slider 14 to support the ball screw with different length. The front bearing seat 5 is mounted on the base body to support the ball screw with back bearing 13 together. The AC servo motor 17 is set on tailstock slider 14 to drive the ball screw move in circle. It will form a position closed loop with long grating encoder 20. So is the linear motor 6 which is installed between the linear guide ways to drive the linear guide slider 10 and air flotation slider 18 moving in a straight line. In the same way, forming a position closed loop with circular grating encoder1, the torque motor 22 is installed on the left side of base body to drive the precision spindle moving in circle. There is a row of mounting holes on one side of base body to have the cantilevers 8 mounted on.

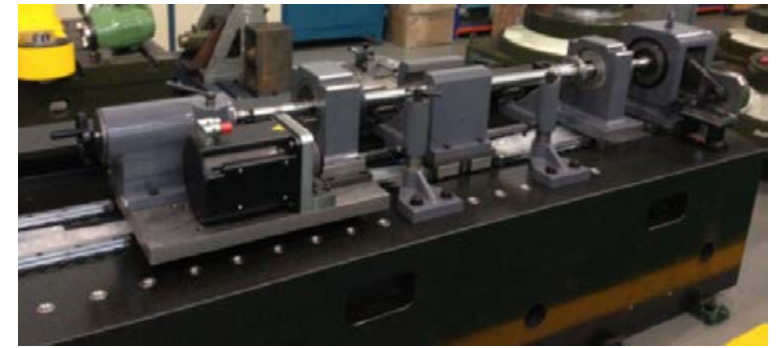

Fig.2 Physical map of ball screw measuring instrument

\section{Test scheme introduction of multi-function ball screw measuring device}

According to the different supporting ways when testing the technical parameters of ball screw, the test bed can be divided into two categories: double dead center test bed and double bearing seat test bed. The former is used to do low speed and high precision test experiments like the measurement of ball screw geometric errors. The latter is used to do high speed and heavy load test experiment like the test of positional accuracy, reverse clearance, speed and acceleration, friction torque, preload, fatigue properties, temperature rise and so on. The former have the characteristics of small vibration and stable performance, but experiments on the latter may be have strong vibration. So it is hard to integrate into one test bed. By the means of reasonable structure design and simply assembly, we can 
conduct these two kinds of test on the ball screw measuring device. In this way, not only the cost is cut down, but also the comprehensive and reliable evaluation for ball screw will be obtained. Aiming at different technical parameters, both the test bed building and experimental schemes will be discussed in details below.

Double dead center test bed. The way of double dead center supporting is taken as benchmark for clamping and machining. Therefore, the way of double dead center supporting has higher measurement precision than the way of double bearing seat supporting. But the dry friction between double dead center and ball screw imposed restrictions on the speed of ball screw, thus, double dead center is the best choice to detect geometric error. Here, double dead center supporting test bed is ball screw geometric error test bed.

Measurement principle and characteristics.

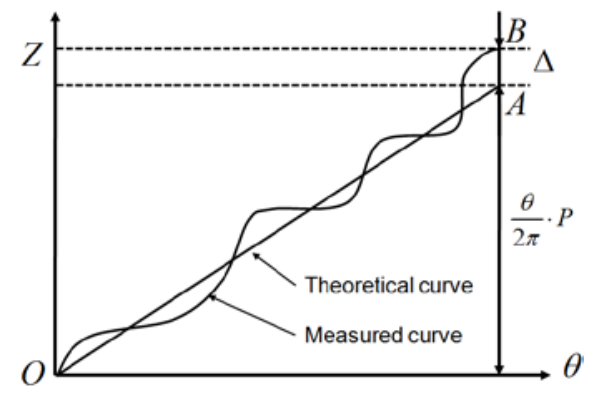

Fig.3 Measurement principle of ball screw lead error

Geometric error measurement of ball screw mainly refers to the lead errors detection, the measurement principle is as shown in figure 3[3]. Based on the absolute value comparison principle of synchronous displacement, take the circular grating encoder and the long grating encoder as the angular and length standard. The torque motor moves in circle at same speed, and linear motor controlled by electronic gear drives the measuring head move in equal proportions [5]. Meanwhile, Angular displacement signal and the axial displacement signal sampled by the high- speed data acquisition card. After the high-expansion electronic division in signal processing system embedded in the data acquisition card, the signals are sent to computer system by PCI bus, and then, convert the signal to displacement, we can obtain the ball screw lead error curve by the real-time processing of computer system. The signals on two channels have nothing to do before data processing. After the computer processing, the signals are converted to angular reference quantity and axial displacement, and error value is the difference between the two values.

Main features of testing:

Based on the principle of electronic gear, the linear motor drives the measuring head moves and generates theoretical helical line with the torque motor's movement of proportion. It is active during measurements, so it has the characteristics of smaller measuring force, higher precision and efficiency than passive measurements.

Compared with the way of "nut drives the measuring frame", it decreases the kinematic errors brought in by ball screw nut assembly, which facilities the later data processing. Meanwhile, there is no dead corner because the nut takes no space in length of ball screw.

Compared with the way of two heads(one driving head and one measuring head), the measuring head has smaller elastic deformation, therefore, there is no elastic deformation error of measuring head during measurement. The measuring frame moves more stably.

High measurement is easier to implement because of small range of measuring head.

Structure design of double dead center test bed.

As is shown in figure 4, it is the double dead center test bed (geometric error test bed) in which the front bearing seat, back bearing seat and nut seat are removed on the basis of overall structure in figure 1. According to the length of ball screw, the positions of tailstock slider can be regulated coarsely in axial direction and fixed on by pneumatic clamps. There is a hand wheel behind the tailstock to adjust finely the supporting distance. The synchronous belt links the precision spindle and torque motor. The fork linking the ball screw and precision spindle can transmit the power from torque motor. According to the length of ball screw and deflection deformation permitted by 
measuring requirement, one or two cantilevers will be used to support the ball screw in the middle, and the support stiffness can be adjusted. Before the measurement, the nut has to be put on the end of no measurement, because the nut can't be disassembled from ball screw. After the measuring part is finished, put the nut on the other end, and go on the measurement of the rest.

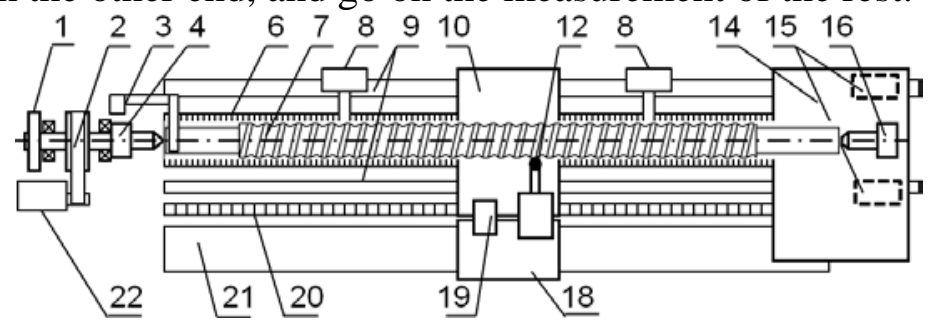

1 circular grating encoder 2 synchronous belt 3 fork 4 precision spindle 6 linear motor 7 ball screw 8 cantilevers 9 linear guide ways 10 linear guide slider 12 measuring head 14 tailstock slider 15 pneumatic clamps16 tailstock 18 air flotation slider 19 linker 20 long grating encoder 21 aerostatic guide way 22 torque motor 23 nut seat

Fig.4 Double dead center test bed structure design diagram

Double bearing seat test bed. The double bearing seat test bed is used for testing some dynamic parameters, like positional accuracy, reverse clearance, speed and acceleration, friction torque, preload, fatigue properties, temperature rise and so on ${ }^{[6-8]}$. These parameters need to be measured at high speed, or with preload or axial force imposed on during measurement. So the requirement of rotation precision is not high, but the requirement of load capacity is higher than geometric error test bed. If we still use the method shown in figure 4, the dead centers could be damaged because of dry friction between the dead center and ball screw at high speed, and the air flotation slider and air flotation block can also be destroyed because of heavy load. In order to keep high precision when detecting the geometric error, the precision spindle and tailstock don't permit to be taken apart. So the two bearing seats set between the two dead centers, and have no location interference with dead centers. The linker between linear guide slider and air floatation slider is disassembled. In this way, the air floatation slider is left alone in one side, and of course, won't be influenced by dynamical characteristics tests ${ }^{[9-10]}$. The double bearing seat test bed structure design diagram is shown in figure 5. It is the basic test bed of dynamical characteristics tests. Some sensors, related testing software and attachments are necessary if certain specific parameters need to be tested.

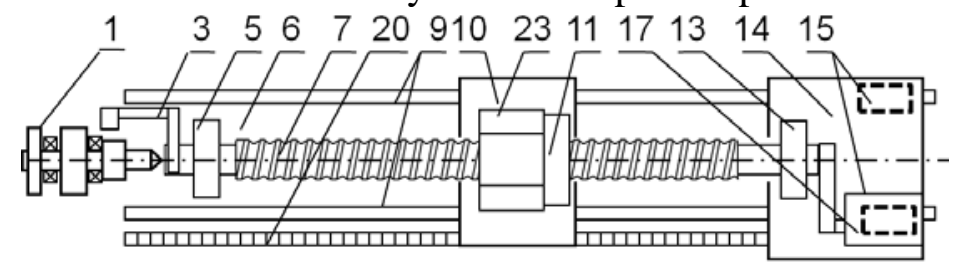

1 circular grating encoder 3 fork 5 front bearing seat 6 linear motor 7 ball screw 9 linear guide ways 10 linear guide slider 11 ball screw nut 13 back bearing seat 14 tailstock slider 15 pneumatic

clamps17 AC servo motor 20 long grating encoder 23 nut seat

Fig. 5 Double bearing seat test bed structure design diagram

Structure design of linker. Fixed on the linear guide slider and air flotation slider, the linker serves two proposes: power transmission and kinematic error reduction of linear guide ways. It transmits drive from linear motor to air flotation slider and measuring head frame. The kinematic precision of linear guide way slider is lower than that of air flotation slider, so the linker has another function of reducing the kinematic errors of linear guide way slider. Figure 6 shows the structure design diagram of linker. The cylindrical body 5 is fixed on the air flotation slider, the rest parts of linker are fixed on the linear guide way slider. The cylindrical body 5 is clamped between plate 3 and column 4, so degree of freedom in direction of motion is limited, but it can move freely in the last two directions (direction perpendicular to paper and direction up or down). There is a group of disc spring 2 inside of the linker, the gripping rigidity can be adjusted by force adjusting knob 1 according to actual working conditions. By this way, the driving problem is settled down without influencing the kinematic precision of measuring head frame. 


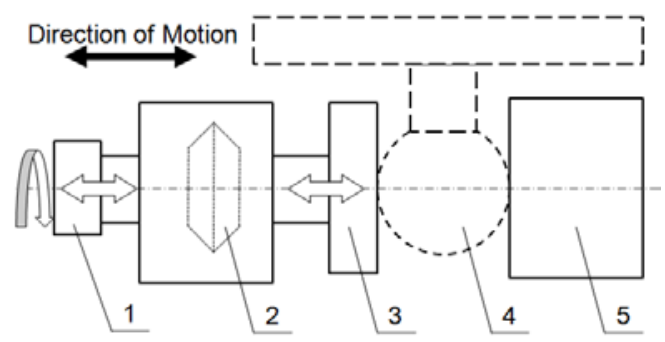

1 force adjusting knob 2 disc spring 3 plate 4 cylindrical body 5 column

Fig.6 Structure Design Diagram of Linker

\section{Conclusions}

In this paper, the majority parameters of ball screw have been analyzed and classified, and a ball screw test bed is introduced. First, according to the characteristics of test parameters, the test bed is divided into two categories: double dead center test bed and double bearing seat test bed. Then, the overall structure design of the test bed is introduced. And the last, the transformation by assembly and disassembly between the two kinds of test bed is introduced. It turns out that the structure design scheme is feasible. The whole test bed has been processed out, after the related test, an accurate and reliable evaluation of the whole performance of ball screw can be obtained.

\section{Acknowledgements}

This research has been supported by China Technology Major Project (NO: 2011ZX04016-031).

\section{References}

[1] Feng Hu-tian, Comprehensive performance measurement method and technology of ball screw Mechanism [M]. Beijing, Mechanical Industry Press, 2011:15-58.

[2] Zhang Shan-zhong, Precision Instrument Structure Design Manual [M], Beijing, Mechanical Industry Press, 2009: 474-497.

[3] Liu Jie-liang, Analysis and Experiment Study on Dynamic Performance of Ball Screw Mechanism [D]. Master Thesis. Xi'an University of Technology, 2005: 8-18.

[4] Feng Hu-tian Li Chun-mei Tao Wei-jun Ou Yi, Error Measurement and Analysis Algorithm of Ball Track of Ball Screw Shaft [J], Journal of Nanjing University of Science and Technology, 2010, 34(5): 575-580.

[5] Zhou Hong-bin, Design of 3-meters ball screw helix error Measurement System based on laser

[D], Master Thesis , Shandong Jianzhu University, 2011:7-14.

[6] Mou Shi-gang, Research on Dynamic characteristic of high-speed Ball Screw with nut Driven [D]. Doctoral Thesis. Shandong University, 2013.

[7] Song Xian-chun, Liu Jian, Wang Zhao-tan, Liu Xian-yin, Li bao-min. Research and Development of Test System of Combination Property of High-Speed Ball Screw Unit [J]. Tool Engineering. 2005,39(3).

[8] Wang Zhao-tan, Liu Xian-yin, Li Bao-min. Test Instrument for Comprehensive Performance of High Speed Precision Ball Screw Units [J]. Manufacture Technology \& Machine Tool. 2007,(5).

[9] Kang Xian-min, Fu Wei-ping, Wang Da-cheng, Wang Jian-sheng. Analysis and Experiment of Effect of Preload Force on Ball Screw Friction Torque Fluctuation [J]. Journal of Mechanical Transmission. 2010, 34(6).

[10] Kang Xian-min. Analysis and Experiment Study on Dynamic Performance of Ball Screw Mechanism [D]. Doctoral Thesis. Xi'an University of Technology. 2011. 